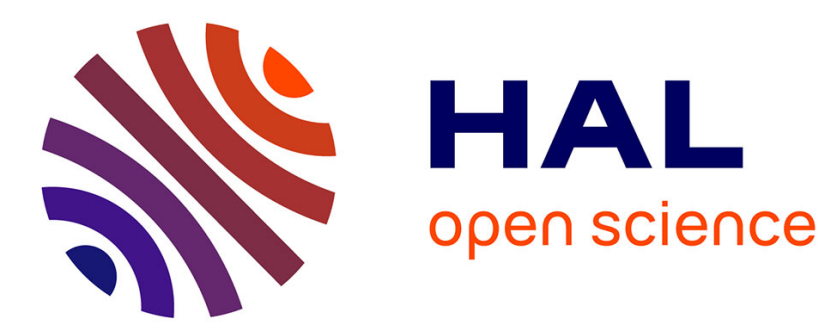

\title{
Nutritional resources as positional information for morphogenesis in the Stony Coral
}

\author{
Michael Kücken, Baruch Rinkevich, Lee Shaish, Andreas Deutsch
}

\section{To cite this version:}

Michael Kücken, Baruch Rinkevich, Lee Shaish, Andreas Deutsch. Nutritional resources as positional information for morphogenesis in the Stony Coral. Journal of Theoretical Biology, 2011, 275 (1), pp.70. 10.1016/j.jtbi.2011.01.018 . hal-00676095

\section{HAL Id: hal-00676095 \\ https://hal.science/hal-00676095}

Submitted on 3 Mar 2012

HAL is a multi-disciplinary open access archive for the deposit and dissemination of scientific research documents, whether they are published or not. The documents may come from teaching and research institutions in France or abroad, or from public or private research centers.
L'archive ouverte pluridisciplinaire HAL, est destinée au dépôt et à la diffusion de documents scientifiques de niveau recherche, publiés ou non, émanant des établissements d'enseignement et de recherche français ou étrangers, des laboratoires publics ou privés. 


\section{Author's Accepted Manuscript}

Nutritional resources as positional information for morphogenesis in the Stony Coral stylophora pistillata

Michael Kücken, Baruch Rinkevich, Lee Shaish, Andreas Deutsch

PII: S0022-5193(11)00030-0

DOI: doi:10.1016/j.jtbi.2011.01.018

Reference: YJTBI 6330

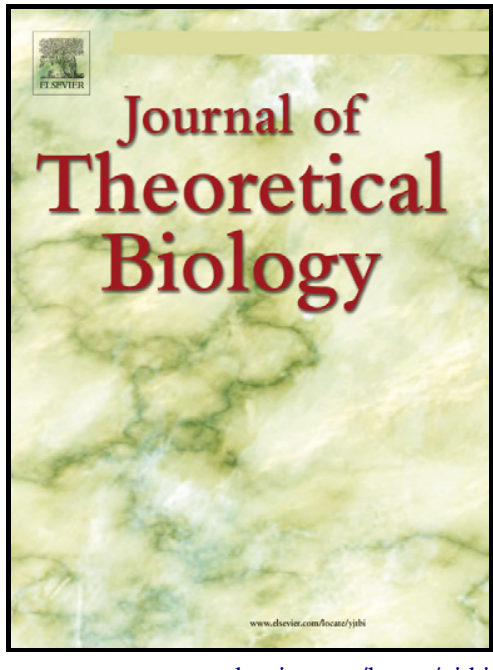

www.elsevier.com/locate/yjtbi

To appear in: Journal of Theoretical Biology

Received date: 10 May 2010

Revised date: $\quad 13$ January 2011

Accepted date: 13 January 2011

Cite this article as: Michael Kücken, Baruch Rinkevich, Lee Shaish and Andreas Deutsch, Nutritional resources as positional information for morphogenesis in the Stony Coral stylophora pistillata, Journal of Theoretical Biology, doi:10.1016/j.jtbi.2011.01.018

This is a PDF file of an unedited manuscript that has been accepted for publication. As a service to our customers we are providing this early version of the manuscript. The manuscript will undergo copyediting, typesetting, and review of the resulting galley proof before it is published in its final citable form. Please note that during the production process errors may be discovered which could affect the content, and all legal disclaimers that apply to the journal pertain. 


\title{
Nutritional Resources as Positional Information for Morphogenesis in the Stony Coral Stylophora pistillata
}

\author{
Michael Kücken ${ }^{1, *}$ \\ Center for Information Services and High-Performance Computing, \\ Technische Universität Dresden, 01062 Dresden, Germany \\ Baruch Rinkevich* \\ Israel Oceanographic and Limnological Research, \\ National Institute of Oceanography, Tel Shikmona, \\ PO Box 8030, Haifa 31080, Israel \\ Lee Shaish* \\ Israel Oceanographic and Limnological Research, \\ National Institute of Oceanography, Tel Shikmona, \\ PO Box 8030, Haifa 31080, Israel \\ Andreas Deutsch* \\ Center for Information Services and High-Performance Computing, \\ Technische Universität Dresden, 01062 Dresden, Germany
}

\begin{abstract}
We are interested in deciphering the mechanisms for morphogenesis in the Red Sea scleractinian coral Stylophora pistillata with the help of mathematical models. Previous mathematical models for coral morphogenesis assume that skeletal growth is proportional to the amount of locally available energetic resources like diffusible nutrients and photosynthetic products. We introduce a new model which includes factors like dissolved nutrients and photosynthates, but these resources do not serve as building blocks for growth but rather provide some kind of positional information for coral morphogen-

\footnotetext{
${ }^{*}$ Corresponding author

Email address: michael@gsystech.de (Michael Kücken )

${ }^{1}$ Present address: Max-Planck Institute for the Physics of Complex Systems, Nöthnitzer Str. 38, 01187 Dresden, Germany
} 
esis. Depending on this positional information side branches are generated, splittings of branches take place and branch growth direction is determined. The model results are supported by quantitative comparisons with experimental data obtained from young coral colonies.

Keywords: coral morphogenesis, mathematical model

\section{Introduction}

Skeletal growth in scleractinian corals occurs by the deposition of calcified matrix which is excreted by the polyp calicoblastic tissue. This process relies on complex interactions between the environment and intrinsic genetic control $[1,2,3,4]$. Two major environmental factors that affect coral growth and morphology are light, that is used as an energy source for the zooxanthellae [5, 6, 7, 8], and water currents [9]. A strong genetic influence is reflected in the bewildering range of shapes that colonies of different coral species can attain. Even within a species genetic factors have a strong influence on morphology [10]. However, very little is known about the genetic control of coral architectures, pattern formation and on the variety of shapes each coral species may present $[4,10,11]$.

In complex biological phenomena such as morphogenesis very often fundamental principles are not very well understood. In this situation mathematical modeling is increasingly considered to be a very useful tool [12]. Through mathematical modeling we can test hypotheses, connect seemingly unrelated observations and establish some order in the confusing plethora of biological observations.

Modeling coral growth has so far focused on the role of environmental factors such as light and water currents. The particular morphology of the coral was proposed to provide an ecological advantage such as better access to light or dissolved nutrients $[13,14]$. In [15] coral tissue is considered analogous to porous media and growth is proposed to be the result of an interaction between water flow and chemical processes.

By far the most elaborate models appeared in a series of papers by Kaandorp, Merks and coworkers $[16,17,18,19,20]$. These studies analyzed the influence of hydrodynamic flow on coral morphology. The coral is modeled as a three-dimensional surface and growth takes place perpendicular to this surface according to a so-called growth function. In the above models the amount of skeletal growth is directly dependent on the concentration of lo- 
cally available nutrients that are transported to the coral by the water flow. Locations of the coral exposed to the water flow will thus exhibit increased growth in contrast to areas that have less access to the water flow. Variations of the model consider diffusion of nutrients inside the coral and the effect of light.

These models succeed in obtaining branching coral architectures that resemble some corals actually found in nature but cannot - as we will argue below - sufficiently describe morphogenesis in the scleractinian coral Stylophora pistillata. This species forms calcified skeletons characterized by well-defined branches that arise through splitting of existing branches and sprouting. In the course of colony astogeny complex, yet not haphazard, branch patterns arise. Note, that morphogenesis by branch bifurcations and sprouting is a common mechanism in morphogenesis - examples are the formation of the tracheal tree in the lung and kidney formation [21, 22].

Stylophora pistillata colonies can be generated either from a small fragment (nubbin) down to the size of a single polyp or from a planula larva that attaches to the ground. At first the tissue around the attached fragment or the primary polyp spreads laterally and new polyps appear as nodes in a seemingly hexagonal lattice. In the case of planula larva settlement, at some point the coral tissue excretes calcified matrix and a branched skeleton is produced. In both scenarios new branches then arise by two mechanisms. First, an existing branch may split into two branches. Second, a new branch may form lateral to an already existing branch — a process that we call sprouting. A quantitative characterization of these first stages of astogeny was given in [10]. We use this data for fine-tuning our model.

Undamaged Stylophora colonies in the wild that are free to grow into all directions usually exhibit a round, almost hemispherical shape. Also, it is observed in young colonies that originate from a single branch that the overall shape is first very elongated and almost planar [10, 23, 24] (see Figure 7 a) and b)) and only later approaches the spherical shape seen in larger colonies. Another important feature of Stylophora is the fact that the branches fill out the colony space quite evenly. Generally, branches avoid each other and gaps in the colony are filled in by new branches [25].

There is a large degree of stochasticity in colony growth. Colonies of the same genotype grown under the same conditions exhibit considerable variation as to the exact location of new side branches and splitting events. The morphometric parameters that were extracted from colonies by Shaish et. al. [10] have some fairly high standard deviations as well. Nevertheless, it 
was shown that differences between genotypes were still larger than stochastic variation within a genotype.

If we observe young colonies that originate from a single separated branch as has been done by Shaish et. al. [10] we notice that significant skeletal growth takes place only at the branch tip and a few locations along the branch giving rise to lateral branches. However, most places of the coral surface exhibit no or very little skeletal growth even though all parts of the branch have equal access to nutrients in the water. This behavior is in contrast to Kaandorp-Merks' model that relates local growth to the amount of locally available resources and predicts initial skeletal growth of the branch on the whole surface in this situation. However, this is not the case in these young colonies and therefore Kaandorp-Merks' model is not suitable for describing morphogenesis in Stylophora pistillata.

In the following we will call the small circular regions that form a significant amount of skeletal matrix growth zones. By definition they are located at a branch tip - but not every branch tip is necessarily a growth zone (if the branch ceased growing). Due to their calcifying activity growth zones likely have a different genetic expression profile than noncalcifying areas. Survival of the growth zone will lead to a continued growth of the branch, a new generation of a growth zone will lead to a lateral branch, a splitting of a growth zone will lead to a branch bifurcation and the extinction of a growth zone will lead to a stop in branch growth.

The lack of significant calcification outside the growth zones indicates that the decision for calcification is not only dependent on the local access to nutrients in the water. Rather, some sort of intracolonial signaling is likely involved. This could for instance be mediated by a substance which is excreted by the growth zone tissue, diffuses to the neighboring tissue and suppresses calcification there. It could also be due to a depletion of resources needed for calcification in the non-calcifying tissue that are transported to the growth zone. The situation we encounter here has parallels to well-known organizing regions such as the Hydra head. Most significantly, formation of a new Hydra head can only take place some distance away from an existing head [26]. Organizing regions have been well described by simple mathematical models that assume a slowly diffusing activator and a faster diffusing inhibitor $[26,27,28]$. In this context the conditions for the emergence and splitting of organizer regions are well-understood. A similar mechanism could well explain the permanence of growth zones and the repression of growth in the neighboring tissue. 
This is not to say that energy resources like nutrients or sunlight are not important for colony growth - quite the opposite, as their involvement is considered axiomatic in the field. However, they likely play a different role in colony astogeny than the one suggested by Kaandorp and Merks. We propose that they influence colony shape not directly as building material for the skeleton but by providing cues for the growth direction of branches and by controlling branch bifurcations and the generation of new branches. In this sense the spatial distribution of energy resources provides positional information to the tissues of the developing colony. The situation is somewhat different to the classical notion of positional information in morphogenesis, which is usually connected to more or less elusive morphogens [29].

In our model we assume that energy resources influence morphogenesis by controlling the survival, disappearance and splitting of growth zones. Generally, high concentrations of resources will lead to the survival, new generation and splitting of growth zones whereas low concentrations will lead to an extinction of a growth zone. An example where nutritional resources indeed have an impact on the emergence of organizing regions is Hydra magnipapillata where overfeeding leads to multiple head formation along the body axis [30]. We assume that energy resources reach the coral from the water medium and are created by photosynthesis. Photosynthates are modeled to be transported inside the coral, which is strongly supported by experiments $[31,32,33]$

Due to the lack of specific information we do not outline a speculative scenario on low-level genetic control but rather include genetic factors in our model parameters. The model considers the coral at the branch level meaning that we do not consider single polyps and the details of branch formation and splittings but rather attempt to understand how colony architecture emerges.

The model results were compared with data on morphogenesis in Stylophora pistillata that were collected by Shaish et. al. [10]. In this work young colonies of several different genotypes of Stylophora pistillata were observed for one year. We used their genotypes A, B and C as these were shown to have significantly different growth parameters and are representative for the other genotypes considered in [10].

\section{Model}

The starting point of our modeling is the realization that significant skeletal growth in Stylophora takes place almost exclusively at the tips of the 
branches. This observation is supported by the fact that branches do not much thicken towards their base. The branch tips are not only interesting as the main sites for calcification but also for some physiological peculiarities. In most Stylophora corals the branch tip is less pigmented which indicates that fewer zooxanthellae are present at the tips. A study of the tips of Acropora revealed that the tips are not only low in zooxanthellae numbers, but also low in photosynthetic products and interestingly very high in ATP [34]. The authors of this study concluded that photosynthetic products are transported from the tissue of the branches toward the tips where they are rapidly converted into ATP. This energy can then aid the calcification process. In Stylophora this kind of study has not been performed but results in $[31,32]$ suggest a similar phenomenon. The observation emphasizes that tissue in different parts of the colony has different functions: the branches produce photosynthetic products that are used up at the branch tip and aid the growth process there. This difference in tasks is likely manifested by a different gene expression profile in the tips. Unlike in plants there is neither apical polarity nor apical dominance in branch growth, a conclusion supported by the fact that isolated branches develop new branches at either end of the branch [24].

Because colony growth takes place predominantly at the branch tips we can represent the coral shape by the midlines of the coral branches. Thus, the coral colony can be viewed as a tree of curves that are growing at the free ends. The curves themselves are modeled as a collection of line segments, all with the same length $\Delta x$, that are attached to each other (see Figure 1 a) b)). The endpoints of the line segments we call nodes and denote them by $\vec{r}_{i}$. This approach reduces the three-dimensional structure of the coral to a collection of one-dimensional objects on which mathematical algorithms perform much faster.

In this representation morphogenesis of the coral is described by the following processes:

- growth of a branch tip

- splitting of a branch tip into two tips

- sprouting of branches on existing branches

As we have outlined in the introduction we propose that the distribution of locally available resources (dissolved nutrients in the water and distribution of photosynthetic products) provides positional information to genetic 
a)

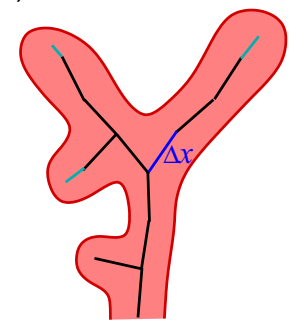

b)

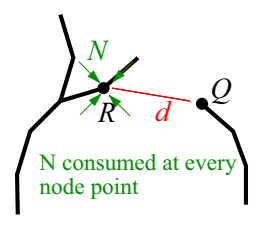

c)

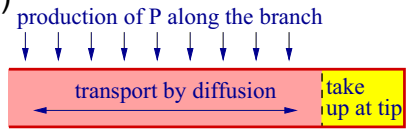

d) high $N$

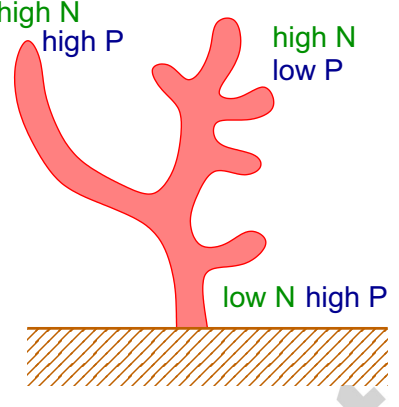

Figure 1: a) The three-dimensional coral skeleton is represented by its midline curve. This curve is itself represented as a collection of line segments, each of length $\Delta x$. Growing line segments are represented in grey. b) Quantity $N$ is taken up at every node $R$, its amount decreases by $\phi(d)$ at a point $Q$ with distance $d$ to $R$. c) We assume that $P$ is produced in the branch, diffuses and is taken up completely at the branch tip. d) The local concentration of $N$ and $P$ provides information about the location within the coral colony. $N$ is large at the periphery and small in the center of the coral. $P$ is large in the center and at long branches but small in areas of many growing branch tips.

factors that guide these processes. For our mathematical model we define two quantities $N$ and $P$ that we interpret as concentrations of nutrients in the water medium and photosynthetic products in the coral branches, respectively. These quantities are used for defining growth direction as well as splitting and sprouting events.

The quantity $N$ represents the concentration of dissolved nutrients in the water current, similar as in Kaandorp and Merks' models [18, 19, 20]. The work of these authors has shown that under broad assumptions the distribution of nutrients in the water medium around the coral is not uniform. Assuming that nutrients reach the coral by advection and diffusion and are taken up by the coral tissue, zones with depleted nutrients arise. Generally, $N$ will be large in the coral periphery and small in the center of the coral (see Figure $1 \mathrm{~d}$ ) and Figure $2 \mathrm{a}$ )). Therefore the magnitude of $N$ provides information to the coral tissue about its relative location in the coral colony.

The movement of nutrients in the sea water is very complicated because advection, diffusion, time-dependent currents, tidal flows and turbulence all play a role. We do not explicitly take these effects into account and define $N$ in a very simple way. We assume that far away from the coral a maximal nutrient concentration $N_{\max }$ is achieved. The coral tissue decreases this maximal nutrient concentration by taking up nutrients itself. We remind that the coral is given by the collection of nodes $\left\{\vec{r}_{i}\right\}$ (the endpoints of the line 
segments), which we use to find $N$ at an arbitrary point $\vec{r}$. Then we assume that every node point $\vec{r}_{i}$ decreases the amount of nutrients present at $\vec{r}$ by $\phi\left(\left|\vec{r}_{i}-\vec{r}\right|\right)$ where $\phi$ is a decreasing function (see Figure $\left.1 \mathrm{~b}\right)$ ). Thus, we have

$$
N(\vec{r})=\frac{N_{\max }-\sum_{i} \phi\left(\left|\vec{r}_{i}-\vec{r}\right|\right)}{N_{\max }} .
$$

In our simulations we used

$$
\phi(r)=\left\{\begin{array}{lll}
\frac{1}{r^{\alpha}} & : & r \geq R \\
\frac{(\alpha+2) R-(\alpha+1) r}{R^{\alpha+1}} & : & r<R
\end{array}\right.
$$

with $\alpha=1.7$ and $R$ is of the order of magnitude of the branch thickness $d$. Function $\phi(r)$ is constructed such that it is continuously differentiable and that it does not diverge for $r=0$. Our choice for $N(\vec{r})$ is motivated by a Green's function approach to the solution of the diffusion equation. Indeed, for $\alpha=1.0$ we have the exact solution of Poisson's equation. Choosing a larger $\alpha$ (as we did) highlights short-distance influences and decreases longdistance influences. Equation (1) does not yet consider the sea floor (which we assume to be planar) on which the coral is growing. We can incorporate the sea floor by extending the sum in (1) to all points $\vec{r}_{i}^{*}$ that are obtained as reflection of $\vec{r}_{i}$ at the sea floor.

According to equation (1) $N(\vec{r})$ can be negative, which is clearly nonbiological. This can be avoided by making $\phi$ dependent on $N$ but this greatly complicates the calculation of $N$. In our model we set $N$ to zero if the sum exceeds $N_{\max }$. This choice ensures a value of $N$ between 0 and 1 . As an alternative for ensuring nonnegative $N$ we could have solved the diffusion equation with sinks that are dependent on the local nutrient concentration, but that would have been computationally significantly more expensive.

The quantity $P$ represents the local availability of photosynthates that are produced in the branches of the coral and are transported through the coral. $P$ is defined on the nodes $\left\{\vec{r}_{i}\right\}$ only. We assume that $P$ is produced evenly by the coral with rate $\lambda$, is completely taken up at the tips (i.e. we have $P=0$ at the tips) and is transported by diffusion (see Figure $1 \mathrm{c}$ )). Therefore, $P$ will be large in the center of the coral and at long branches with no side branches. It will be small in areas with many growing branch tips (see Figure $1 \mathrm{~d}$ ) and Figure $2 \mathrm{~b}$ )).

Additionally, we assume that there is a maximal concentration of photosynthates $P_{\max }$. Due to scaling arguments we can set $P_{\max }=1$. Thus, values 

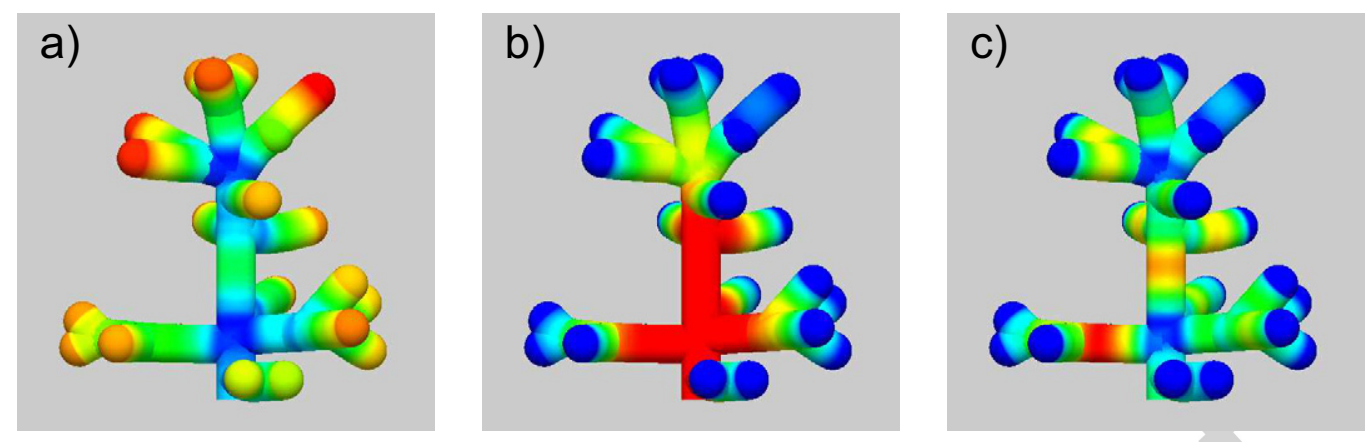

Figure 2: Concentrations of a) $N$ b) $P$ and c) sprouting probability in a colony of genotype $\mathrm{C}$ after 100 simulation steps. Red/yellow corresponds to large values and blue/green to small values. $N$ is small in the center of the coral and large at the branch tips. $P$ is large in the center and small in the periphery. The largest probability for a sprout is found at the left lower branch and in the middle of the main branch.

for $P$ are in the interval from 0 to 1 . On a particular branch, $P$ is given by the steady-state diffusion equation

$$
P_{x x}+\lambda=0
$$

where $P=0$ at branch tips, $P_{x}=0$ at the base of the coral tree and $P$ is conserved at branch nodes. The equation is solved using a straight-forward iterative procedure. If after an iteration step $P$ exceeds $P_{\max }$ it is set to $P_{\max }$.

Let us now continue with the rule for growth at the branch tip. We assume the same growth speed $v$ for all growing tips. Unfortunately, there are no long-term measurements of the growth speed of individual branches although experiments for settling this question would be easy to perform. Assuming a constant growth rate is still well-supported in face of the spherical growth of colonies. Also we observe after a splitting event that the two new branches have about the same length.

Note that it frequently happens in corals that branch tips stop growing. This is often the case for interior branches, and may be due to the lack of access to dissolved nutrients from the flow and lack of light. Apparently, the disposition to growth is switched off completely in these cases. This behavior was partly incorporated in the model. Growth of a branch was stalled if it was to collide with another branch or with the surface on which the coral grows. For the small colonies that were mostly considered the effect was not important.

For the growth direction of a branch we use the gradient of $N$ (direction 
of largest increase in $N$ ) measured at the tip of the branch. This way growth of the branches is generally directed away from the colony center as it is actually observed in virtually all colonies. Furthermore, this rule reproduces the fact that branches avoid and grow away from each other. The rule is supported by observations in [25]. It was shown that branches whose tips are placed close to each other grow away from each other or stop growing at all. This property of the coral branches to grow away from each other is certainly one of the main reasons why the coral fills out space evenly with its branches. We do not include phototropism in our model because it does not seem to play a primary role for determining the growth direction in the young colonies that we observed [24].

Sprouting branches are important to fill in space for colonies that originate from a single branch such as in the experiments of $[10,23,24]$. In many young colonies that originate from a separated branch the number of sproutings significantly exceeds the number of branch splittings. We assume that sprouting takes place at a certain location in a given iteration step with a probability $S(N, P)$ which increases as resources $N$ and $P$ increase. For simplicity we assume that $S(N, P)=\beta N P$. This choice for $S(N, P)$ implies that sproutings only occur in areas where neither $N$ nor $P$ are too small. It ensures that interior branches fill out the space between existing branches and that new branches do not form in areas that are already nutrient-depleted. Other, slightly different choices for $S(N, P)$ give similar results. Note that our rule is not deterministic but stochastic, which takes into account the large variability that is observed during the first stages of colony formation.

The direction of a new sprout is not directly given by the gradient of $N$. Instead, points in different directions are sampled and the direction in which the largest $N$ was found is used as the direction of the branch. This direction is in some cases very different from $\nabla N$.

Branch splitting is the common mechanism for the generation of new branches in larger colonies but also occurs frequently in the young colonies investigated in [10]. Observations suggest that the two new branches split with an angle of about $35^{\circ}$ although variations do occur in real specimens. We assume that the two new arising branches are located in a single plane with the branch from which they originate and that the new branches are symmetric around the axis of the old branch. This assumption is well-supported by observations. The last rotational degree of freedom is determined by the position of the new branches that maximizes the sum of $N$ on the tip of the two branches. Splittings are modeled to occur randomly with probability 
of $T(N, P)=\gamma N+\delta P_{x}$. Here $P_{x}$ is the derivative of $P$ along the branch and proportional to the flux of $P$ into the tip. Note that this implies that splitting occurs if $N$ is large (meaning that the branch is well-exposed to the fluid flow) or $P_{x}$ is large (meaning that the branch is very long already with no side branches).

Of course, other forms for the probabilities $S(N, P)$ and $T(N, P)$ could be used. Our choices are simple, do not contain many parameters and give good results.

We continue with a summary of our main model assumptions:

- the coral grows exclusively at the tips with a fixed growth rate

- the coral consumes dissolved nutrients $N$ from the water along the branches

- photosynthetic products are produced along the branches, diffuse and are taken up completely at the branch tips

- branches grow into the direction of the steepest decrease of $N$ into the water medium

- side branches sprout randomly with a probability proportional to the product of $N$ and $P$

- branch tips split randomly with a probability proportional to the sum of $N$ and $P$

In our simulations we start with a single branch growing upward with length $H_{\text {init }}$. This is in analogy to the experiments reported in [10]. After the initialization a number of iteration steps is performed. At each step the following calculations take place:

- increase the segment of the growing tips by a fixed amount

- if the segment is longer than $\Delta x$ start a new line segment but give a chance for splitting and calculate the new growth direction using $\nabla N$

- calculate $P$ and $N$ on the branches

- give a chance for sprouting at every node point

- calculate $P$ again if sprouting took place 


\begin{tabular}{|l|l|}
\hline Parameter & Explanation \\
\hline $\mathrm{H}$ & colony height \\
$\mathrm{nB}$ & number of branches \\
$\mathrm{N} 2$ & number of branches of order 2 \\
$\mathrm{~N} 3$ & number of branches of order 3 \\
$\mathrm{Le}$ & colony length \\
& largest lateral extension \\
$\mathrm{Ev}$ & ecological volume \\
& $\pi H r^{2}$ where $r=$ widthlength/4 \\
$\mathrm{SI}$ & percentage of branches from splitting \\
$\mathrm{Bal}$ & average branch length \\
$\Omega$ & largest order of a branch \\
\hline
\end{tabular}

Table 1: List of morphometric parameters that were used in the quantitative comparison of experiments and simulations. Refer to details of how the parameters were measured to [10].

\begin{tabular}{|l|l|l|l|}
\hline parameter & genotype A & genotype B & genotype C \\
\hline$v(\mathrm{~mm} / \mathrm{step})$ & 0.0875 & 0.144 & 0.144 \\
$H_{\text {init }}(\mathrm{mm})$ & 28.6 & 28.0 & 32.0 \\
$\beta$ & 0.01 & 0.004 & 0.01 \\
$\gamma$ & 0.02 & 0.00625 & 0.02 \\
$\delta$ & 0.02 & 0.00875 & 0.02 \\
\hline
\end{tabular}

Table 2: Choice of model parameters for genotype A, B and C.

For the small colonies that we consider the algorithm is very fast, but it greatly slows down for larger colonies.

After a simulation run the morphometric quantities of coral growth in Table 1 were extracted. We strove for consistency in the computation of these quantities with [10]. For each genotype 30 simulation runs were performed and averages and standard deviations of the morphometric quantities were computed and compared to the corresponding values found experimentally [10].

The visualization tool TRIVIZ written by H. Gerdes was used for graphic output. This tool is a $\mathrm{C}++$ interface to the more general OpenGL and allows an efficient visualization of three-dimensional structures. 

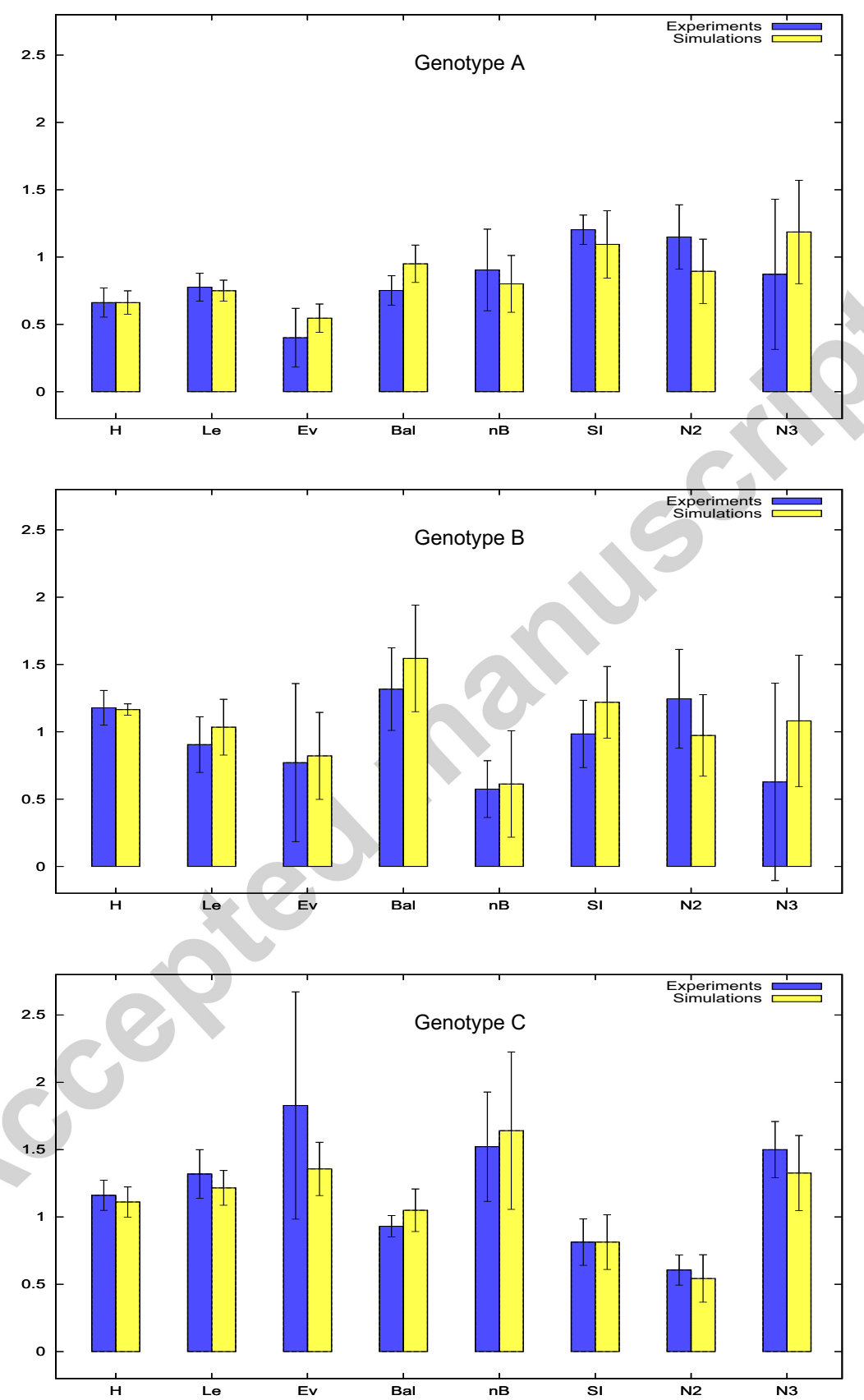

Figure 3: Comparison of experiments and simulations. The data are scaled to the average of the experimental data for the three genotypes. 


\begin{tabular}{|l||l|l||l|l||l|l|}
\hline parameters & A exp & A sim & B exp & B sim & C exp & C sim \\
\hline $\mathrm{nB}$ & $16.7 \pm 5.6$ & $14.8 \pm 3.9$ & $10.6 \pm 3.9$ & $11.3 \pm 7.3$ & $28.1 \pm 7.5$ & $30.3 \pm 10.8$ \\
$\Delta \mathrm{H}(\mathrm{mm})$ & $44.5 \pm 2.1$ & $45.5 \pm 1.0$ & $56.0 \pm 11.4$ & $56.3 \pm 1.0$ & $59.5 \pm 8.6$ & $58.7 \pm 2.1$ \\
$\mathrm{~N} 2(\%)$ & $72 \pm 15$ & $56 \pm 15$ & $78 \pm 23$ & $61 \pm 19$ & $38 \pm 7$ & $34 \pm 11$ \\
$\mathrm{~N} 3(\%)$ & $25 \pm 16$ & $34 \pm 11$ & $18 \pm 21$ & $31 \pm 14$ & $43 \pm 6$ & $38 \pm 8$ \\
$\mathrm{Ev}\left(\mathrm{cm}^{3}\right)$ & $24.3 \pm 13.2$ & $33.1 \pm 6.4$ & $46.8 \pm 35.6$ & $49.8 \pm 19.6$ & $110.9 \pm 51.2$ & $82.3 \pm 12.0$ \\
$\mathrm{SI}(\%)$ & $77 \pm 7$ & $70 \pm 16$ & $63 \pm 16$ & $78 \pm 17$ & $52 \pm 11$ & $52 \pm 13$ \\
$\mathrm{Le}(\mathrm{mm})$ & $30 \pm 4$ & $29 \pm 3$ & $35 \pm 8$ & $40 \pm 8$ & $51 \pm 7$ & $47 \pm 5$ \\
$\mathrm{Bal}(\mathrm{mm})$ & $7.6 \pm 1.1$ & $9.6 \pm 1.4$ & $13.3 \pm 3.1$ & $15.6 \pm 4.0$ & $9.4 \pm 0.8$ & $10.6 \pm 1.6$ \\
$\Omega$ & $3 \pm 1$ & $3.5 \pm 0.6$ & $3 \pm 1$ & $3.3 \pm 0.7$ & $4 \pm 1$ & $4.8 \pm 0.9$ \\
\hline
\end{tabular}

Table 3: Quantitative comparison of simulations and experiments for genotypes A, B and C.

\section{Results}

Three sets of parameters were fitted by hand corresponding to three genotypes (genotype A, B and C in the experiments of [10]). For all simulations we used $\Delta x=0.125 \mathrm{~cm}, \alpha=1.7$ and $\lambda=22.0, N_{\max }=12.9$ and a splitting angle of $36^{\circ}$. 100 simulation steps correspond to one year. The remaining parameters for each genotype are given in Table 2. The initial colony height $H_{\text {init }}$ was chosen as the average initial height in the experiments for the respective genotype.

Snapshots of a typical simulation run for genotype $\mathrm{C}$ are presented in Figure 6. The same simulation, but seen from above and for more iterations, is shown in Figure 7 c)-f). As in experiments we observe a planar shape of the coral and later a transition to a more rounded, spherical shape. A large colony corresponding to three years development is seen in Figure 5. This colony exhibits a spherical shape and qualitatively resembles corals in the field.

Comparisons between simulations and observed colonies for genotypes A, $\mathrm{B}$ and $\mathrm{C}$ are shown in Figure 4. Clearly, a resemblance between experiments and simulations is visible. Exact correspondence cannot be expected because of the large component of stochasticity in colony development.

In Table 3 morphometric parameters of experiments and simulations are compared. The same data are presented visually in Figure 3. In spite of the sometimes large error bars each genotype has a distinct profile of morphometric parameters that has been reproduced by the simulations.

In our simulation runs we observed that height, the number of branches, 

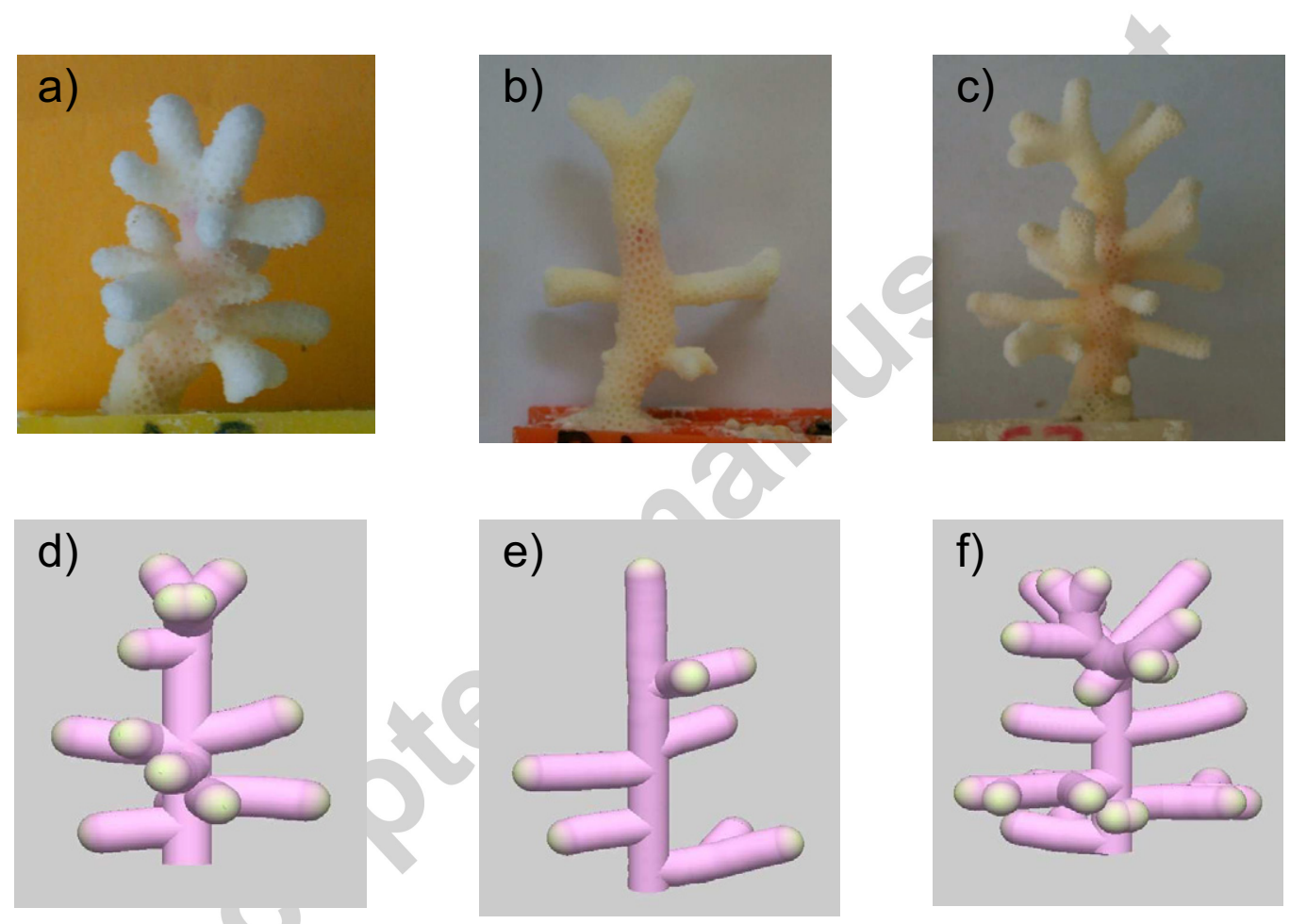

Figure 4: Comparison of experiments and simulations for genotypes A a), d) B b), e) and C d), f) 


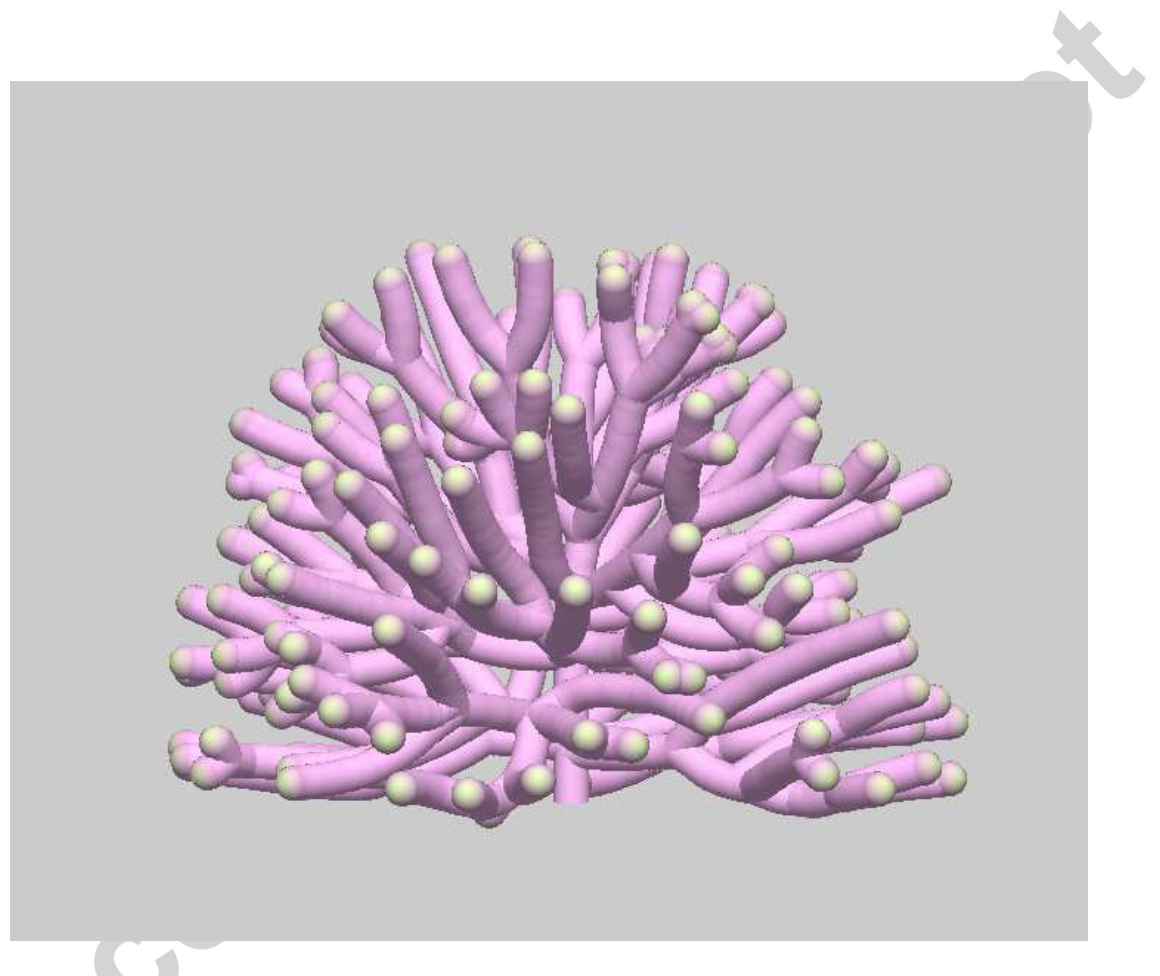

Figure 5: Simulation for genotype C after 300 steps corresponding to development after three years. 

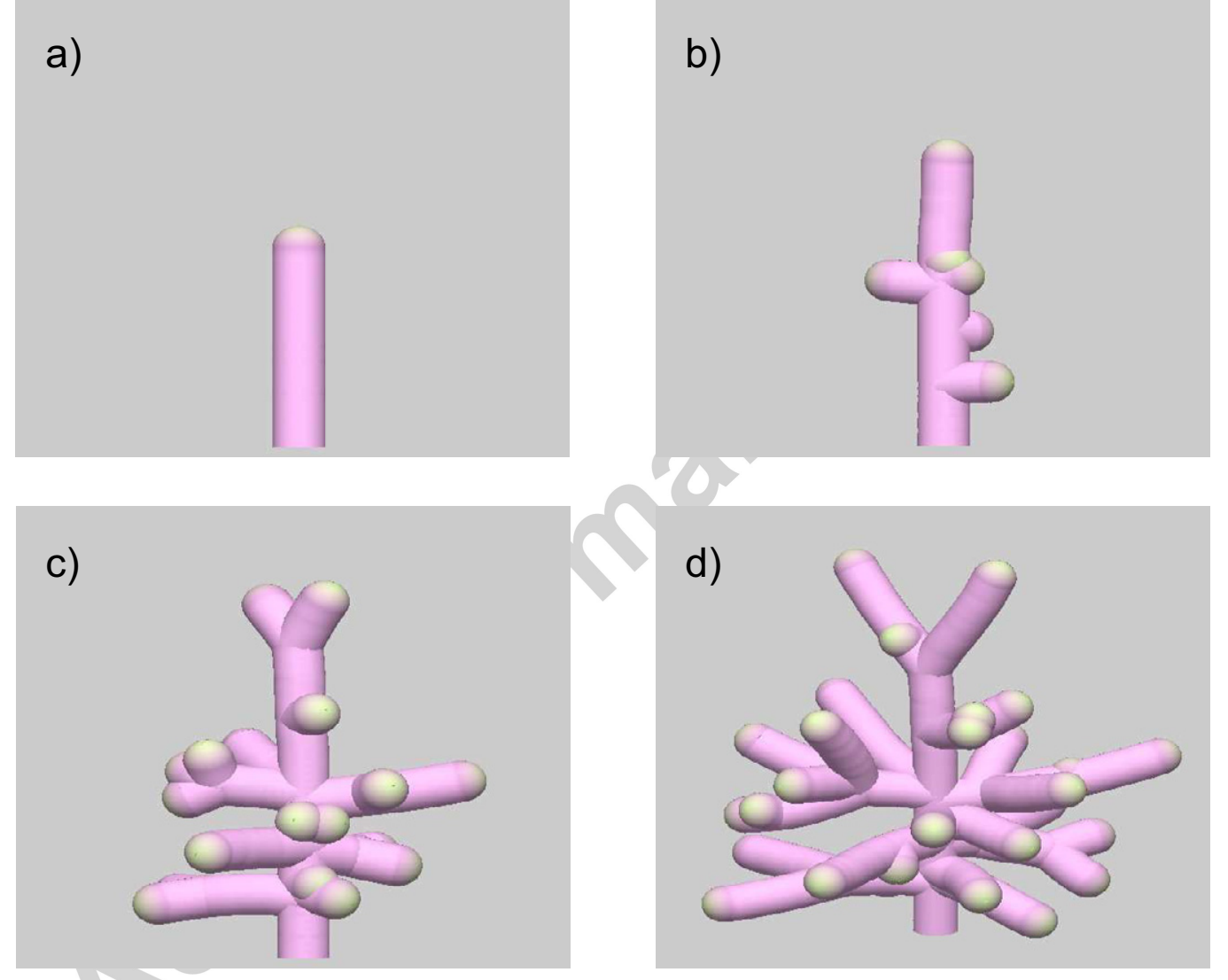

Figure 6: Simulation for genotype C after a) 0 steps b) 50 steps c) 100 steps and d) 150 steps. 100 time steps correspond to one year. 

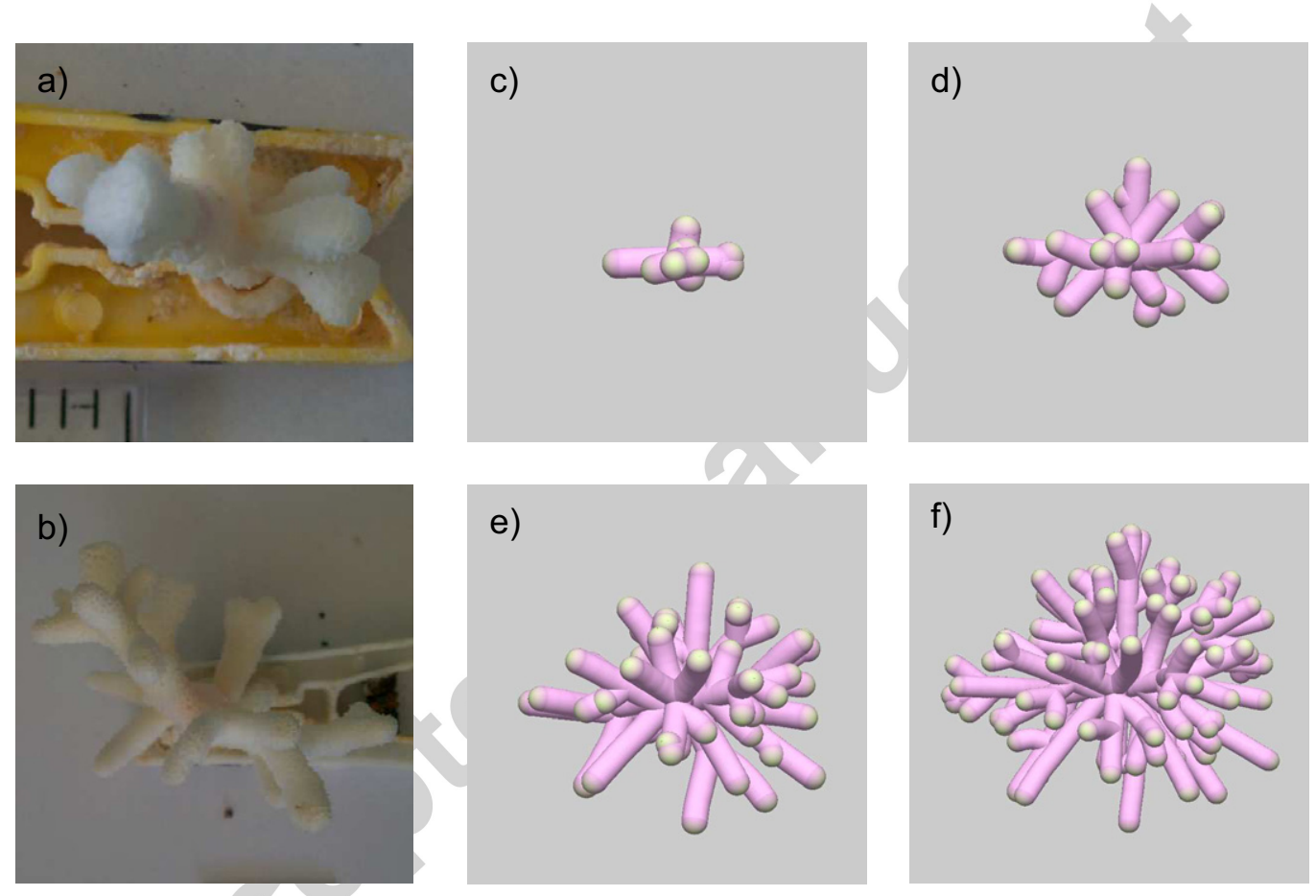

Figure 7: Colonies after one year seen from the top of genotype a) A and b) C. Simulation for genotype $\mathrm{C}$ seen from the top after c) 50 steps d) 100 steps e) 150 steps and f) 200 steps. 100 time steps correspond to one year. 
ecological volume and length could be very well reproduced. The simulation data are almost always in the confidence interval of the experimental data. The average branch length is consistently too large in the simulations which may be due to slightly different definitions of branch length. Furthermore, the largest order of branches $\Omega$ in the simulations is usually too large and N2, the number of branches of order 2, is too small. Again, a different technique in determining order is likely to be the culprit for this difference.

Note that not only the averages correspond well to each other but also the standard deviations of some parameters. In both experiments and simulations for the same settings quite different colonies were obtained which supports our view that there is a large stochastic component in the beginning of astogeny.

Along the environmental factors there is a large genetic influence on morphogenesis. We observed that genotype A has a much smaller growth rate than the other two genotypes and that genotype B has far fewer branches than genotypes A and C. Genotype A also has fewer branches than genotype $\mathrm{C}$, which is due to the slow growth because the splitting and sprouting probabilities are actually the same in A and C. This means that splitting and sprouting probabilities are not dependent on the growth rate. Small colonies can already exhibit an intricate and complex branched structure (like genotype A) and, vice versa, relatively large colonies can still have relatively few branches (like genotype B).

There is not only quantitative agreement between simulations and experiments but also qualitative agreement in features that are more difficult to quantify. The visual appearance of the simulated structures resembles real corals. The branches in the simulations seek out to fill the space around the coral. Furthermore, the beginning of astogeny is characterized by a planar colony shape, exactly as it is actually observed (see for example Figure 4a) and $\mathrm{b})$ ).

\section{Discussion}

Our model not only qualitatively reproduces the early stages of coral development but also compares very well quantitatively to the experimental data. This is the more remarkable as measuring the morphometric parameters occurs by very different tools in experiments and simulations. Due to the one-dimensional nature of the model, calculations can be executed very efficiently and many runs can be performed easily and fast. 
We postulate the following hypotheses regarding coral morphogenesis in Stylophora:

- dissolved nutrients and the distribution of photosynthates provides positional information for coral morphogenesis

- coral growth takes place at the branch tips only

- growth at the branch tips is uniform

- sprouting and splitting are stochastic events that are regulated by the presence of dissolved nutrients and photosynthates

Crucial for the model is the realization that significant skeletal growth only takes place in one zone - at the branch tip. Sprouting can be understood as the formation of a new growth zone and splittings can be seen as a large growth zone that is dividing into two growth zones. If a growth zone has been established we propose that the growth rate is constant for all growing tips. If the tip should encounter an area of few resources growth is halted completely as it is in fact frequently observed in inner branches of the colony with poor access to the flow around the coral. In our model we have not yet considered this feature as it is not relevant for young colonies.

The idea of the growth zone can be used to understand growth in different coral species. In a branching coral like Stylophora pistillata the growth zone has the shape of a disk. How this shape is established is not part of our model but could be addressed by a model that represents the coral as a surface in three dimensions - possibly by a reaction-diffusion mechanism that is regulated by the availability of resources. Note that reaction-diffusion patterns can also be line patterns which might underlie the linear growth zones of some corals such as Turbinaria and some Montipora species.

There are similarities in our approach to the model by Kaandorp and Merks by emphasizing the role of energetic resources such as solved nutrients and photosynthates. However, energetic resources play a different role in our model compared to Kaandorp-Merks' model. In their model skeletal growth was proportional to the amount of locally available nutrients. In our model nutrients control the emergence and splitting of growth zones. Skeletal growth does not take place outside of growth zones even though nutrient concentration might be high. In Kaandorp-Merks' model one observes a continuous range of growth speeds along the coral. In our model there is either growth at a constant speed or no growth at all. 
The model also has similarity to a classic model by Meinhardt for the development of branched structures [35]in tissues. One version of the model consists of an activator $A$ that leads to differentiation in the tissue. This activator is paired to a classic inhibitor $I$ that prevents the occurrence of a new activator center in the near vicinity and a diffusible substance $S$ that is guiding the developing branch away from already differentiated tissue. Linear structures with branch bifurcations and lateral branches arise in the course of a simulation. Although the formulation of Meinhardt's model is different and it is applied to differentiation, not growth, its logic is very similar to ours. The activator $A$ and the differentiation process correspond to the branch tip that is growing at a constant speed. The substance $S$ corresponds to our $N$ in that it is guiding the branch - and the inhibitor $I$ corresponds to both $N$ and $P$ in that it is controlling the formation of side branches. There is no explicit inhibitor in our model but inhibition occurs by depletion of the resources $N$ and $P$.

Our model does not downplay the importance of genetic factors, which are contained implicitly. For instance, the maintenance of a relatively small, circular growth zone at the branch tip requires a genetic basis. In our model we do not address how this growth zone is established but we point out that circular dot patterns are ubiquitous in nature and can be the result of many different mechanisms. Furthermore, the way positional information is used for determining growth direction, splitting and sprouting probabilities necessarily requires a genetic basis. We do not outline the details of this process, which would be pure speculation only with the lack of knowledge on genetics in Stylophora pistillata.

It is important to note that the introduced quantities $N$ and $P$ can be interpreted differently. Instead of $N$ we could have introduced the quantity

$$
I(\vec{r})=\sum \phi\left(\left|\vec{r}_{i}-\vec{r}\right|\right),
$$

as a representation of an inhibitor of growth that is excreted by the coral branches. The mathematical structure of such a kind of model is very similar to our model and it is in fact very difficult to propose an experiment that discriminates between the two scenarios. Similarly, $P$ could also be interpreted in a different way. Instead of a quantity taken up at the tips and promoting sprouting and splitting we could define a substance that is excreted at the tip and represses sprouting and splitting along the branch. Again, it is impossible with current knowledge to decide what mechanism is 
correct. Therefore we do not rule out in our model the possibility that the coral regulates its own growth by true morphogens that are acting through the branches and the water medium. On the other hand there is strong evidence that dissolved nutrients and photosynthetic products are not distributed evenly so that they can serve as positional information, and there is no necessity for morphogens that do the same job.

Finally, it appears that some of the presented ideas can be used to find more general growth rules for a wider class of coral species. Especially, the idea that growth takes place in certain growth zones only may also apply to other coral species.

\section{Acknowledgements}

This research was funded by the European project 012547 CORALZOO. We thank Heiko Gerdes (Dresden) for the visualization of the model results. We thank the editor for useful remarks that greatly improved the paper.

[1] J. P. Gattuso, M. Frankignoulle, I. Bourge, S. Romaine, R. W. Buddemeier, Global Planet Change 18 (1998) 37-46.

[2] J. P. Gattuso, D. Allemand, M. Frankignoulle, Amer Zool 39 (1999) 160-183.

[3] F. Houlbreque, E. Tambutte, D. Allemand, C. Ferrier-Pages, J Exp Biol 207 (2004) 1461-1469.

[4] B. Rinkevich, Isr J Zool 48 (2002) 71-82.

[5] P. G. Falkowski, Z. Dubinsky, Nature 289 (1981) 172-174.

[6] M. Hidaka, S. Shirasaka, J Exp Mar Biol Ecol 157 (1992) 69-77.

[7] B. Rinkevich, Y. Loya, Mar Biol 80 (1984) 1-6.

[8] B. Rinkevich, Mar Biol 101 (1989) 259-263.

[9] M. A. Reidenbach, S. G. Monismith, J. R. Koseff, A. Yahel, G.and Genin, Limnol Oceanogr 51 (2006) 1956-1968.

[10] L. Shaish, A. Abelson, B. Rinkevich, Dev Dyn 235 (2006) 2111-2121. 
[11] P. A. Todd, Biol Rev 83 (2008) 315-337.

[12] J. T. Tomlin, D. A. Axelrod, Nature Rev Gen 8 (2008) 331-340.

[13] R. R. Graus, I. G. Macintyre, Science 193 (1976) 895-897.

[14] K. Kim, H. Lasker, J Exp Mar Biol Ecol 215 (1997) 49-64.

[15] S. Mistr, D. Becovici, Ecosystems 6 (2003) 61-74.

[16] J. A. Kaandorp, C. P. Lowe, D. Frenkel, M. A. Sloot, Phys Rev Lett 77 (1996) 2328-2331.

[17] J. A. Kaandorp, Mar Biol 134 (1999) 295-306.

[18] J. A. Kaandorp, P. M. A. Sloot, J Theor Biol 209 (2001) 257-274.

[19] R. M. H. Merks, A. G. Hoekstra, J. A. Kaandorp, S. P. M. A., J Theor Biol 224 (2003) 153-166.

[20] R. M. H. Merks, A. G. Hoekstra, J. A. Kaandorp, P. M. A. Sloot, J Theor Biol 228 (2004) 559-576.

[21] P.-T. Chuang, A. P. McMahon, Trends Cell Biol 13 (2003) 86-91.

[22] A. M., Z. R., C. E., Nature Rev Mol Cell Biol 10 (2009) 831-842.

[23] L. Shaish, A. Abelson, B. Rinkevich, PloS one 2 (2007) e644.

[24] L. Shaish, B. Rinkevich, PloS one 4 (2009) e4095.

[25] B. Rinkevich, Y. Loya, Bull Mar Sci 36 (1985) 319-324.

[26] H. Meinhardt, Dev Biol 157 (1993) 321-333.

[27] M. Meinhardt, The Algorithmic Beauty of Sea Shells, Springer, 4th edition, 2009.

[28] M. Meinhardt, Models of Biological Pattern Formation, Academic Press, 1982.

[29] L. Wolpert, J Theor Biol 25 (1969) 1-47.

[30] M. Kroiher, Int J Dev Biol 43 (1999) 817-821. 
[31] B. Rinkevich, Y. Loya, Coral Reefs 1 (1983) 243-247.

[32] B. Rinkevich, Y. Loya, J Exp Mar Biol Ecol 73 (1983) 175-184.

[33] U. Oren, Y. Benayahu, H. Lubinevsky, Y. Loya, Ecology 82 (2001) 802 813.

[34] L. Fang, Y. J. Chen, C. Chen, Mar Biol 103 (1989) 359-363.

[35] H. Meinhardt, Differentiation 6 (1976) 117-123. 